\title{
INTER-DIVISION IX-X-XI WORKING GROUP ASTRONOMY FROM THE MOON
}

\section{PRESIDENT \\ VICE-PRESIDENT PAST PRESIDENT MEMBERS}

\author{
Sallie L. Baliunas \\ Yoji Kondo \\ Norio Kaifu \\ Oddbjørn Engvold, Norio Kaifu, \\ Haruyuki Okuda, Yervant Terzian
}

TRIENNIAL REPORT 2006 - 2009

\section{Introduction}

During the period the Working Group had proposed and was granted renewed status by Division XI Space and High-Energy Astrophysics. Additionally the Working Group requested to be extended to Division IX Optical and Infrared Techniques, Division X Radio Astronomy, as well as Division XI .

We recall the memory of a member of the Organizing Committee Willem Wamsteker († 24 November 2005). Norio Kaifu, past chair of the Working Group, is appreciated for his past outstanding service.

Haruyuki Okuda had previously remarked, in the Business Meeting of the WG at the IAU XXVI General Assembly in Prague, 2006, that new opportunities are emerging across the world for lunar research. Haruyuki Okuda's remarks remain in the forefront of scientific goals. We are mindful of Haruyuki Okuda's parallel comment, namely, that such projects necessarily will be large in scope and infrastructure, requiring international cooperation.

\section{Working Group Business Meeting at the IAU XXVII GA in 2009}

Interested participants were asked to discuss and generate proposals for the 2009 IAU General Assembly concerning astronomy from the moon.

A Business Meeting that will include scientific reports has been requested to be scheduled at the IAU XXVII General Assembly in Rio de Janeiro, Brazil, August 2009.

The website <www.cfa.harvard.edu/moon> is being re-structured for the distribution of WG material.

Sallie L. Baliunas

chair of the Working Group 